PALEO

Revue d'archéologie préhistorique

$18 \mid 2006$

Varia

\title{
Les pièces esquillées : état des connaissances après un siècle de reconnaissance
}

Splintered pieces: our state of knowledge after a century of research

Die ausgesplitterten Stücke: Wissensstand nach einem Jahrhundert Forschung

\section{Foni Le Brun-Ricalens}

\section{OpenEdition}

Journals

Édition électronique

URL : http://journals.openedition.org/paleo/181

DOI : 10.4000/paleo.181

ISSN : 2101-0420

\section{Éditeur}

SAMRA

\section{Édition imprimée}

Date de publication : 1 décembre 2006

Pagination : $95-114$

ISSN : 1145-3370

Référence électronique

Foni Le Brun-Ricalens, «Les pièces esquillées : état des connaissances après un siècle de reconnaissance », PALEO [En ligne], 18 | 2006, mis en ligne le 01 septembre 2009, consulté le 07 juillet 2020. URL : http://journals.openedition.org/paleo/181 ; DOI : https://doi.org/10.4000/paleo.181

\section{(c) (i) (2)}

PALEO est mis à disposition selon les termes de la licence Creative Commons Attribution - Pas d'Utilisation Commerciale - Pas de Modification 4.0 International. 


\title{
LES PIÈCES ESQUILLÉES : ÉTAT DES CONNAISSANCES APRÈS UN SIĖCLE DE RECONNAISSANCE
}

\author{
Foni LE BRUN-RICALENS ${ }^{(1)}$
}

\begin{abstract}
Résumé : Cette contribution a pour objet de dresser un état de la recherche sur les pièces esquillées. Suite à un siècle d'investigations, elle tente de faire le point des connaissances à la lumière des investigations pratiquées ces demières décennies en technologie, en tracéologie et en ethnologie d'une part, et des résultats issus d'expérimentations d'autre part. Après un historique rappelant les diverses dénominations anciennement émises, une définition des pièces esquillées est proposée. Ensuite sont présentées et discutées les différentes interprétations formulées quant à l'obtention et à l'utilisation des pièces esquillées, à savoir : outils, nucléus ou déchets?
\end{abstract}

Mots-clés : Industrie lithique, pièces esquillées, technologie, typologie, tracéologie, ethnologie, archéologie expérimentale, outils, nucléus, déchets.

Keywords: Lithic industry, splintered pieces, technology, typology, traceology, ethnology, experimental archaeology, tools, cores, waste products.

\section{Zusammenfassung}

DIE AUSGESPLITTERTEN STÜCKE: WISSENSSTAND NACH EINEM JAHRHUNDERT FORSCHUNG. Dieser Beitrag beschäftigte sich mit dem Forschungsstand der Ausgesplitterten Stücke. Es wird einerseits der Versuch unternommen, den Wissenstand der letzten Jahrzehnte betreffend Technologie, Spurenanalyse und Ethnologie zu beleuchten und andererseits die aus der experimentellen Erprobung hervorgegangenen Resultate aufzuzeigen. Nach Darstellung der Forschungsgeschichte mit den verschiedenen in der Vergangenheit gebräuchlichen Bezeichnungen, wird eine Definition der Ausgesplitterten Stücke vorgeschlagen. Anschließend werden verschiedene Interpretationsmöglichkeiten betreffend der Erzeugung und Nutzung der Ausgesplitterten Stücke aufgeführt und diskutiert: Gerät, Kern oder Abfall?

Schlüsselwörter: Steinindustrie, Ausgesplitterte Stücke, Technologie, Typologie, Spurenanalyse, Ethnologie, experimentelle Archäologie, Gerät, Kern, Abfall.

\section{Abridged english version}

\section{SPLINTERED PIECES: OUR STATE OF KNOWLEDGE AFTER A CENTURY OF RESEARCH}

Recognized at the beginning of the $20^{\text {th }}$ century, splintered pieces remain a particular category of prehistoric lithic artefacts, which, depending on the criteria employed, are sometimes considered as cores, and sometimes as tools or waste. After a century of resear$\mathrm{ch}$, and in light of studies conducted in the last decades, this paper presents a brief historic review and documentary summary of our knowledge of these objects, followed by a critical review and revision of the archaeological, typological, technological, traceological, ethnological and experimental data.

The term "pièce esquillée" seems to have been proposed by the Abbots L. Bardon, J. and A. Bouyssonie in 1906. Later, other denominations were employed in the Francophone literature to qualify the variants of this artefact type (outil esquillé-écaillélsplintered-scaled tool, ciseau/chisel, gouge, coin/wedge, chasse-lame/punch, bigome/anvil, éclateur/flaker, outil-bar/bar-tool etc.), and in the AngloSaxon literature to describe "splintered pieces" (scaled and splintered flake, slotting tool, bifacial lozenge, wedge). Following the generalization of typological classifications during the second half of the $20^{\text {th }}$ century, these different terms were regrouped under theneutral designation: "pièce esquillée" (Brézillon 1983). 


\section{Typo-technological data}

From a typological point of view, a splintered piece is an artefact that generally has a quadrangular form and is frequently splintered, sometimes bifacially, on its two opposing extremities (fig. 1), and more rarely on one or four of them. The shock wave lines of the "splinter" are often very marked and closely spaced. The splinters on one or two faces (inferior, superior, other) can be short, hinged, invasive, or even overshot. The edge, particularly the one that has been struck, is generally bevelled. This irregular or regular, straight or curved "edge cusp" can be altered by crushing and smashing. The splinters are not the products retouching, but rather removals that present stigmata of percussion/utilization. It is not unusual to observe splintered pieces with more or less pronounced traces of longitudinal fracturation, called "éclats d'esquillé" (splinter flake). These fracturations can sometimes detach a bladelet with a triangular or trapezoidal section similar to a burin spall, called a "lamelle d'esquillé" (splinter bladelet) (Demars and Laurent 1992).

From a technological point of view, splintered pieces are generally realized on varied blank categories: small block, flake/blade, flake/blade fragment, retouched or not. Diverse short or elongated quadrangular forms, on flake or blade blanks (or fragments), were employed depending on the raw materials available and the debitage techniques employed at the sites. The numerous, closely compacted shock waves observable on the splinter scars suggest that the splintered pieces were struck with force. J. Tixier recognized three stages:

1 - beginning of splintering on one edge,

2 - removal of splinter flakes, invasive, sometimes bifacial, splinters, and utilization of the other edges,

3 - intensive utilization leading to the fragmentation of the piece; detachment of flakes and/or "splinter bladelets", later also called "bâtonnets"(Tixier 1963).

The diverse technological stigmata described can result both from indirect bipolar percussion or direct percussion, though this latter hypothesis is rarely proposed.

\section{Experimental data}

Indirect bipolar percussion

A splintered piece is easily obtained with a hard stone billet by striking vertically in the axis of the object while it is held with its narrow side against a more or less compact and resistant material. The artefact, employed as a core or intermediary piece, is maintained vertically at the beginning of the operation, with the material-support (to be worked or not) placed on another support (leg, ground, other). After several vertical strikes in the axis of the object, "splinters", resembling "split" fractures, are formed on one or two faces. The majority of the splinters initiate from the edge that is struck, but also sometimes from the other extremity by repercussion, in particular if the inactive material is hard, such as a stone anvil.

Whatever the orientations employed, a splintered piece with splinters located principally on the edge that is struck is obtained. The "cusp" of this edge often rapidly becomes very sharp and can curve inwards depending on either the percussion and utilization angle (perpendicular or oblique), or the morphology of the piece. Therefore, splintered pieces produced on an artefact with a dissymmetrical profile, such as a scraper with abrupt retouch, will tend to splinter first on the inferior face. The removals on the superior face (in this case, a scraper front) function as a "back/striking platform" until the "morphological balance" of the symmetrical plane of the two splintered faces of the piece is "re-obtained" (fig. 2). Therefore, considering the constraints imposed by physical laws, the "axis of percussion" will rapidly tend to be confused with the "axial plane of morphological symmetry".

\section{Direct percussion}

After selecting a blank that is sufficiently large and heavy to be hafted (insertion and maintenance with ligature, etc.), the results are similar to those described above, except that it is now the striking edge that is preferentially splintered.

\section{Traceological data and experimentation}

Splintered pieces are poorly adapted to microscopic use-wear analysis since the contact between the intemediary piece and the material worked is often very brief, thus leaving few traces on the artefact. In addition, the splinters produced during utilization rapidly remove the potential traces from the piece. Nonetheless, the information contributed by the microscopic analysis of splintered pieces indicates that during the Palaeolithic, Mesolithic, and Neolithic, these objects were used for the debitage and working of hard organic materials (bone, antler, ivory, wood), or even mineral materials. It also shows that the splintered edge was not forcibly the active edge of the object.

In an experimental study, splintered pieces (fig. 6) were used to work organic materials. In the context of fragmentation techniques, we observed that the longitudinal fracturation of organic materials (bone, antler, ivory, wood) can be achieved more rapidly with a splintered piece than by sawing with a burin. However, this latter technique is more precise and less random.

\section{Interpretations: archaeological and ethnographic data}

The most common interpretation of splintered pieces is that they served as cores and/or intermediary pieces for indirect bipolar percussion. They thus present use traces created by violent percussion and a morphology resulting from more or less prolonged utilization. Core hypothesis: The use of certain splintered pieces as bipolar cores to produce small, more or less elongated, removals has been demonstrated at some Palaeolithic sites, in particular the Barbera Caves (I) where the debitage is similar to the "split" fracturation tech- 
nique, and at the sites of Buraca Grande, Buraca Escura and Salto do Boi (P). It has also been proposed for some European Epipaleolithic, Mesolithic, and Neolithic sites, as well as for certain African, and North and South American assemblages.

Ethnographically, such techniques are known in Australia where numerous flakes obtained by bipolar percussion are hafted onto the points of spears called "death-spears".

Intermediarypiece hypothesis: A splintered piece - hafted or not - can be used as an intermediary "tool" to fracture, split, divide, fraction, rough-out, notch, bore, cut, etc., through bipolar or direct percussion. In bipolar percussion, two categories, "wedge" or "gouge" types, can be distinguished depending on the working angle (perpendicular or oblique). With direct percussion, two other categories, "adze" and "axe" types, can also be distinguished depending on the fixation (perpendicular or oblique) of the "cutting edge" relative to the handle.

Ethnographically, artefacts similar to splintered pieces, called "sinew-frayeurs" are employed in Africa to split organic materials (animal and/or vegetal), and notably to "divide vine fibres" (Leakey 1931).

\section{Archaeological and chronological considerations}

In archaeological research over the last decades, spatial analyses of lithic and osseous industries, as well as the systematic practice of refitting $(3,4$ et 5$)$, have led to a renewal of paleoethnological approaches (fig. 6). These behavioural analyses (still rare), favoured by dynamic observations of refits or close relations between splinters and splintered pieces, tend to show for the Paleolithic an immediate utilization of the latter, particularly near hearths, as at Hauterive-Champréveyres (CH) (Leesch 1997). Moreover, at certain Aurignacian sites, such as Beauville-Hui (F), a relation with certain billets with "cupules" has been proposed (Le Brun-Ricalens 1989, 1996), but is not systematic.

In the Palaeolithic, Magdalenian sites offer the most significant documents for understanding certain techniques for the fracturation and shaping of animal bones. Ivory, a very resistant material, could have been worked using similar procedures (Otte 1997; Hahn 1995; Khlopatchev 2002). During the Mesolithic, the use of an intermediary piece for splitting was recognized among the different techniques used to fracture antler (David 2002). During the Neolithic, bone, antler and wood working develops significantly alongside a diversification of tools, particularly hafted tools for specialized activities. In conjunction with the development of agricultural productions, new tools were also fabricated, in particular those used to hull grains. Among these, certain elements present similarities with splintered pieces, such as the lithic objects inserted by percussion into a wood support to fabricate a tribulum.

\section{Documentary review: splintered pieces...}

The status of splintered pieces remains, depending on the periods, sites, regions and activities, difficult to comprehend; diverse finalities being obtainable with a similar technique. Most often, it is an integration of technological, traceological and archaeological analyses of lithic assemblages that allows us to clarify the intentions of the prehistoric artisans: core and/or intermediary piece, this latter category including "real" tools as well as "a posteriori tools", and waste.

\section{...cores, tools and waste}

The great recurrence of splintered pieces within prehistoric industries can be explained by the facility with which they can be produced, requiring both minimal skill and minor technical investment. In addition, they offer multiple possibilities and combinations, making them complementary objects appreciated throughout prehistory for their versatility, in both nomadic and sedentary societies.

\section{1 - INTRODUCTION}

Reconnues au début du XX⿳亠丷⿵冂丶 demeurent une catégorie particulière d'artefacts au sein des industries lithiques préhistoriques. En fonction des critères employés (notions d'ordre taxonomique, approches technomorphologique et fonctionnelle, etc.), elles sont tantôt considérées comme des nucléus, tantôt comme des outils ou des déchets. Après un siècle de recherche, à la lumière des investigations menées ces demières décennies, cette contribution propose de dresser un bilan des connaissances avec un rappel et une révision critique des données archéologiques, typologiques, technologiques, tracéologiques, ethnologiques et expérimentales.

\section{2 - HISTORIQUE DE L'APPELLATION}

Le terme " pièce esquillée " ${ }^{2}$ semble avoir été proposé la première fois par les abbés Lucien Bardon, Jean et Amédée Bouyssonie en 1906 dans la publication portant sur les fouilles qu'ils effectuèrent sur le site paléolithique de la Coumba-del-Bouïtou ${ }^{3}$ en Corrèze, France (Bardon et al. 1906 p. 170-175). À cette occasion, ils décrivent également un processus d'obtention expérimenté sur la fouille (Bardon et al. 1906 p. 170, 1908 p. 32) avant les travaux publiés par L. Coutier (1929).

Par la suite, plusieurs autres dénominations, parfois combinées ensemble (ex : " ciseau-gouge "), sous-entendant géné-

(2) qu'ils appellent également " pièce écaillée" (Bardon et al. 1906 p. 171).

(3) également orthographié Coumbo-del-Bouïtou. 
ralement des fonctions particulières, ont été employées dans la littérature pour qualifier et décrire des variantes de ce type d'artefact : " retouchoir" (Leakey 1931 p. 174, " outil esquilléécaillé " (Breuil 1932 p. 8), " ciseau " ", " gouge ", " coin ", " chasse-lame ", " bigome " (Cheynier 1934 p. 305), " éclateur " (Octobon 1938 p. 412), " pièce à extrémité martelée " (Tixier 1954 p. 96), " refendoir ", " feuillardier " (Saumagne 1957 p. 470-471), “ éclat écaillé " (Heinzelin 1962 p. 22), " outil-bar" (Cheynier 1963 p. 147), etc. Ces différents termes peuvent être regroupés sous l'appellation neutre : " pièce esquillée " (Brézillon 1983 p. 288). Divers travaux anglosaxons (MacDonald 1968 ; Lothrop 1982 ; Frison 1988 ; Gramly 1990) utilisent pour les "splintered pieces "également d'autres noms : "scaled and splintered flake ", "slotting tool ", " bifacial lozenge ", " wedge ".

Suite à la généralisation des classifications typologiques dans la seconde moitié du XXème siècle (Le Brun-Ricalens 2005 p. 2372), diverses définitions ont été proposées pour caractériser cette catégorie d'artefact (en particulier Sonneville-Bordes et Perrot 1956 p. 552 ; Müller-Beck 1957-58 ; Heinzelin 1962 p. 22 ; Tixier 1963 p. 146 ; Crémilleux et Livache 1976 ; Fiedler 1979 ; Binder 1987 p. 75 ; Pelegrin 1988 p. 367 ; Demars et Laurent 1992 p. 94-95 ; Hahn 1993 p. 248-251). Les pièces esquillées font régulièrement l'objet de débats qui mêlent des considérations d'ordre typologique, technologique, tracéologique, pouvant inclure des données archéologiques, ethnographiques et expérimentales (par exemple White 1968 ; Escalon de Fonton 1969 ; Newcomer et Hivernel-Guerre 1974; Mazière 1984 ; Chauchat et al. 1985 ; Eickhoff 1988 ; Le Brun-Ricalens 1989 ; Beugnier 1997 ; Lucas et Hays 2004).

\section{3 - APPROCHE DESCRIPTIVE ET DÉFINITION TYPOLOGIQUE}

Artefact généralement de forme quadrangulaire présentant fréquemment sur deux extrémités opposées (fig. 1), plus rarement sur une ou les quatre, des esquillements, parfois " bifaciaux" 5 , dont les ondes de choc sont souvent bien marquées avec des rides serrées. Les esquillements se développant sur un ou deux versants (face inférieure, supérieure, autre), peuvent être aussi bien courts, rebroussés, qu'envahissants, voire outrepassés. Le bord, en particulier celui qui a été percuté, présente généralement un biseau. Ce " fil tranchant ", au délinéament irrégulier ou régulier, droit ou courbe, peut être altéré par des " écrasements mâchurés ".

Ces esquillements ne sont pas des retouches, mais des enlèvements présentant des stigmates de percussion/utilisation. II n'est pas rare d'observer des pièces esquillées présentant des traces de fracturation orthogonales plus ou moins importantes (" éclats d'esquillé ", Demars et Laurent 1992 p. 95). Ces fracturations peuvent parfois détacher sur le bord une lamelle de section triangulaire ou trapézoïdale assez proche de la lamelle coup de burin, appelée " lamelle d'esquillé " (Demars et Laurent 1992 p. 95) (cf. également paragraphe 3.1, stade 3 d'utilisation), voire un éclat " janus " (Newcomer et Hivernel-Guerre 1974 p. 124-126 ; Tixier et al. 1980 p. 90) (fig. 4, $n^{\circ} 3$ et 6) proche d'un petit " déchet Kombewa " (Inizan et al. 1995 p. 73 ; Tixier et Turq 1999).

\section{4 - APPROCHES TECHNOLOGIQUES ET EXPÉRIMENTALES}

\section{1 - Données technologiques}

Les pièces esquillées ont été réalisées sur différentes catégories de support : petit bloc, éclat/lame, fragment de lame/éclat, retouché ou non, voire des petits nucléus recyclés (ausgesplitterte Kerne, Hahn 1988, pl. $32 n^{\circ}$ 6). Ont été sélectionnés de préférence aux produits " d'intention première " (Pelegrin 1986), des supports de " second choix" (Pelegrin op. cit.), des sous produits issus de différentes phases de la ou des chaînes opératoires mise(s) en œuvre ou des outils (usés, cassés, etc.) recyclés (Bardon et Bouyssonie 1906 p. 170 et 171).

Divers modules quadrangulaires courts ou allongés, sur éclat ou (fragment de) lame, peuvent être rencontrés. Ces modules reflètent le "style " des industries réalisées en fonction des matières premières disponibles et des techniques de débitage employées sur les gisements (Brézillon 1968 ; Rozoy 1968 ; Lenoir 1975 ; Tixier et al. 1980 ; Demars 1986).

Les nombreuses rides serrées observables sur les négatifs d'esquillements suggèrent que les pièces esquillées ont été percutées avec force (Tixier 1963 p. 147), généralement à l'aide d'un matériau dense constitué de grains à très forte cohésion (Pelegrin 2000 p. 79-80).

Trois stades ont été reconnus :

1 - début d'esquillement sur un bord,

2 - détachement d'éclats esquillés, esquillements envahissants, parfois " bifaciaux" " , et utilisation d'autres bords,

3 - emploi intensif entrainant la fragmentation de la pièce ; avec l'obtention d'éclats ou/et de "lamelles d'esquillé " (Demars et Laurent 1992 p. 95, $n^{\circ} 10$ ) (fig. 1, n 8), encore appelées " bâtonnets " (Tixier 1963 p. 147). Ces enlèvements proches des " accidents Siret " (Bordes 1961 p. 32) évoquent parfois certaines chutes du burin de Corbiac (Bordes 1970a p. 108), ainsi que des "pseudo-burins " et " outils a posteriori" (Bordes 1970b p. 200-201).

Les divers stigmates technologiques décrits ${ }^{6}$ peuvent provenir aussi bien d'une percussion indirecte posée que

(4) à notre connaissance, terme employé pour la première fois en 1730 par Mahudel (Hamy 1906).

(5) par " bifaciaux ", entendre " esquillements affectant les faces supérieure et/ou inférieure " (Tixier, in litteris).

(6) afin de faciliter les comparaisons, lors des illustrations, il est utile de figurer les pièces esquillées de profil et/ou en coupe (Tixier 1963). 

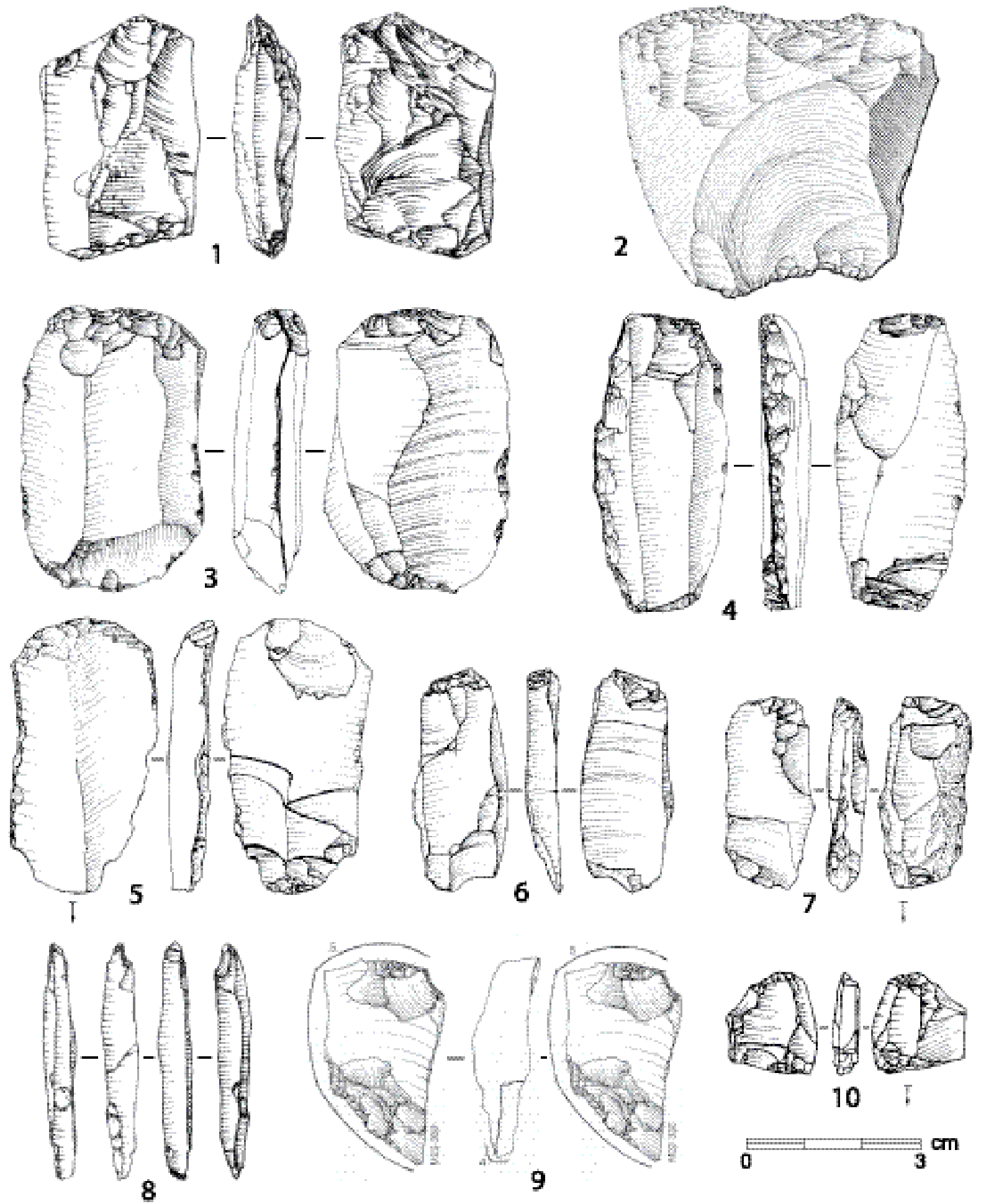

Figure 1 - Exemples de pièces esquillées de différents modules provenant de différents sites préhistoriques. 1 et 8 : Coumba-del-Bouitou (d'après Laurent et Demars 1992) ; 2 : Le Piage (d'après Champagne et Espitalié 1981); 3 et 4 : Vogelherd couche IV (d'après Hahn 1977) ; 5, 6, 7 et 10 : Geissenklösterle (d'après Hahn 1988) ; 9 : HauteriveChampréveyres (d'après Cattin 2002).

Figure 1 - Examples of splintered pieces of varied sizes from different prehistoric sites. 1 and 8: Coumba del Bouitou (after Laurent and Demars 1992); 2: Le Piage (after Champagne and Espitalié 1981); 3 and 4: Vogelherd couche IV (after Hahn 1977); 5, 6, 7 and 10: Geissenklösterle (after Hahn 1988); 9: Hauterive-Champréveyres (after Cattin 2002). 


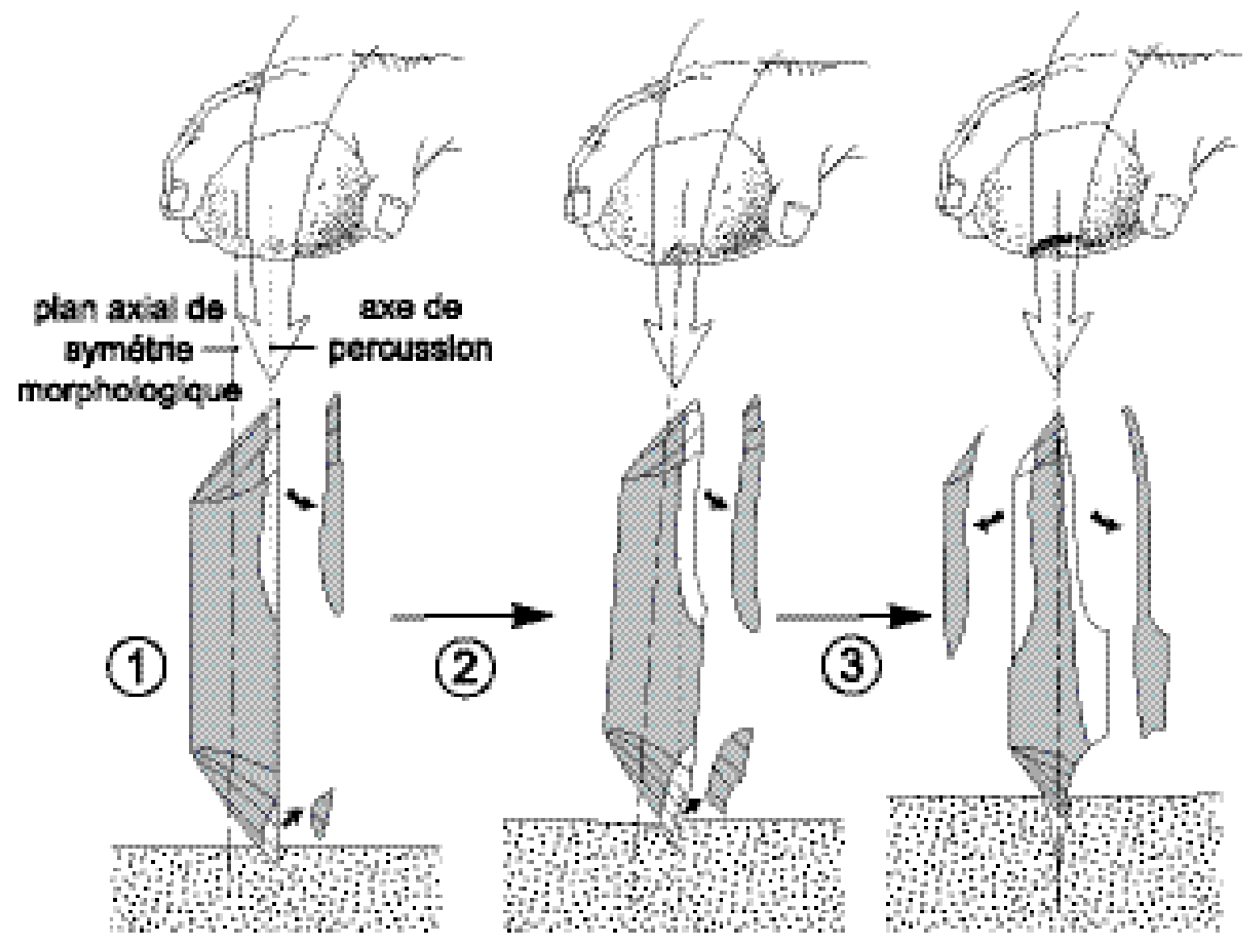

Figure 2 - Différentes étapes d'une "reprise d'équilibre morphologique " (esquillement préférentiel de la face inférieure) d'une pièce esquillée obtenue sur un outil, en l'occurrence un grattoir double, au sens typologique, (d'après Le Brun-Ricalens 1989).

Figure 2 - Different stages of the "reworking of the morphological equilibrium" (preferential splintering of the ventral face) of a splintered piece realized on a tool, in this case a double scraper, in the typological sense (after Le Brun-Ricalens 1989).

d'une percussion directe lancée, bien que cette demière hypothèse soit rarement évoquée (Rigaud 1977). La première catégorie comprend deux types d'utilisation non exclusifs l'un de l'autre, à savoir un emploi comme " nucléus " ou comme " pièce intermédiaire ". La seconde peut nécessiter une fixation/insertion à un manche.

L'hypothèse anciennement proposée pour le débitage indirect, proposant l'utilisation d'une pièce esquillée comme " chasse-lame" en pierre (Cheynier 1934 p. 305) ne semble pas recevable (Tixier 1963 p. 146). De plus, à notre connaissance, aucun exemple ethnographique employant ce type de procédé n'est actuellement connu.

\section{2 - Données expérimentales}

Percussion indirecte posée

Une pièce esquillée est obtenue aisément avec un percuteur minéral dur en frappant verticalement dans l'axe d'un artefact posé de champ sur un support plus ou moins compact et résistant. L'artefact, employé comme nucléus ou pièce intermédiaire, est tenu au début de l'opération entre les doigts ou avec une baguette fendue, tandis que le matériau-support (à travailler ou non) est posé sur un appui (jambe, sol, autre). Après plusieurs coups verticaux dans l'axe du support, il se forme alors rapidement, sur une ou deux faces, des esquillements évoquant les fractures en "split " partant en plus grande partie du bord frappé, mais aussi parfois de l'autre extrémité posée par contre coup (Barham 1987), en particulier si le matériau dormant est dur, telle une enclume en pierre.

Quelles que soient les orientations utilisées, on obtient une pièce qui s'esquille principalement sur le bord qui est percuté (Tringham et al. 1974 ; Le Brun-Ricalens 1989). Le " fil " de ce bord, souvent rapidement " coupant ", peut s'incurver en fonction de l'angle de percussion et d'utilisation (perpendiculaire ou oblique) mais aussi en fonction de la morphologie de la pièce (Migal 1987). Ainsi, les pièces esquillées exécutées sur un artefact à p rofil dissymétrique tel un grattoir à retouches abruptes auront tendance à s'esquiller tout d'abord sur la face 
inférieure $^{7}$ (Rigaud 1977) évoquant les " grattoirsgouges " (Nelissen 1956 p. 49 ; Brézillon 1968 p. 240) et les " ciseaux" (Bordes 1967 p. 51). En effet, les enlèvements présents sur la face supérieure (front du grattoir en l'occumence) jouent le rôle d'un " dos/plan de frappe " jusqu'à la " reprise d'équilibre morphologique " du plan de symétrie entre les deux faces d'esquillement (fig. 2). De ce fait, eu égard aux contraintes qu'imposent les lois physiques, "l'axe de percussion " tendra rapidement à se confondre avec le "plan axial de symétrie morphologique " (Le Brun-Ricalens 1989 p. 199).

\section{Percussion directe lancée}

Comme précédemment après avoir sélectionné un support suffisamment grand et lourd pour pouvoir être emmanché (insertion ou maintien par ligature, etc.). Les résultats sont similaires à ceux décrits ci-dessus, hormis le fait que c'est le bord percutant qui s'esquille préférentiellement (Rigaud 1977 ; Caspar et al. 1998 ; Brenet et al. 2001).

\section{5 - APPROCHES FONCTIONNELLES}

L'usage des pièces esquillées demeure difficile à appréhender car il semble recouvrir diverses activités. Parallèlement à la production de supports lithiques (hypothèse nucléus), le façonnage de matières dures périssables (bois végétal et animal, os, ivoire, etc.) souvent évoqué (hypothèse outil), est confirmé par les quelques données tracéologiques disponibles (Gassin 1996 ; Beugnier 1997 ; Lucas et Hays 2004).

\section{1 - Données tracéologiques}

Les pièces esquillées se prêtent mal à l'étude des microtraces d'utilisation. Le contact entre la pièce intermédiaire et la matière travaillée pouvant être bref, les traces sont par conséquent peu nombreuses sur l'artefact. De plus, les esquillements provoqués par l'utilisation ôtent rapidement les éventuelles micro-traces (Semenov 1964).

Néanmoins, les informations apportées par ce type d'examen microscopique sur des pièces esquillées indiquent, tant pour le matériel paléolithique (Keeley 1980 ; Vaughan 1985a et b ; Symens 1988 ; Christensen 1999 ; Hays et Lucas 2001 ; Plisson et Vaughan 2002 p. 95 ; Lucas et Hays 2004), mésolithique (Rodriguez à paraître), que celui néolithique (Cahen et al. 1986 ; Caspar 1988 ; Beugnier 1997), un débitage et un travail de matières organiques dures (os, bois - animal et végétal -, ivoire), voire minérales (Brenet et al. 2001), et le fait que le bord esquillé ne soit pas forcément le bord actif de l'objet (Chauchat et al. 1985 ; Lucas et Hays 2004).

\section{2 - Interprétations : hypothèses de travail, données archéologiques et ethnographiques}

L'interpétation la plus courante des pièces esquillées est celle d'un nucléus et/ou d'une pièce intermédiaire de percussion indirecte posée présentant des traces d'utilisation par percussion violente, dont la morphologie résulte d'un emploi plus ou moins prolongé (voir paragraphes 2 et 3 ).

\subsection{1 - Nucléus à esquilles}

L'emploi de certaines pièces esquillées comme nucléus bipolaires (bipolar scalar core) destinés à fournir des petits enlèvements plus ou moins allongés a été, d'une part, démontré pour certains sites paléolithiques, en particulier dans les grottes de Barbera (I) (Cancellieri et al. 2001) où le débitage est proche de la fracturation en "split " (D'Angelo et Mussi 2005), sur les sites de Buraca Grande, de Buraca Escura et du Salto do Boi (P) (Aubry et al. 1997), et, d'autre part, proposé pour des gisements épipaléolithiques (Orliac et Orliac 1973) et mésolithiques européens (Aubry et al. 1998 ; Zilhão et al. 1997 p. 293-295), mais aussi néolithiques (Guyodo et Marchand 2005), ainsi que pour des séries africaines (Gobert 1958 p. 40 ; Tixier 1963 ; Barham 1987 ; Jones 1994 ; Villa et al. 2005 p. 413-415), nord(Patterson et Sollberger 1976 ; Cresson 1977 ; Haynes 1977 ; Russel-Stafford 1977 ; White 1977 ; Hayden 1980 ; Schott 1999) et sud-américaines (Chauchat et al. 1985 p. 38 ; Mansur-Franchomme 1986).

Ce type de production (percussion posée sur enclume en tapant verticalement dans l'axe du support) effectuée souvent à partir de matériau de très bonne qualité, permet d'obtenir de nombreux produits fins et coupants, éclats et lamelles d'esquillé dont le gabarit est néanmoins difficile à standardiser.

Sur le plan ethnographique (Schott 1989), de tels procédés de "nucléus à esquilles " sont connus notamment en Nouvelle-Guinée (White 1968) et en Australie (Kamminga 1971). De nombreux éclats obtenus par percussion bipolaire sont fixés sur des pointes de sagaie appelées " death-spears " (Chauchat et al. 1985). Ces armes évoquent certains rares exemplaires archéologiques paléolithiques attribués au Magdalénien (Allain et Descouts 1957) et mésolithiques (Rust 1943 ; Taute 1968 ; Rozoy 1978 ; Nuzhnuyj 1989).

\subsection{2 - Pièce intermédiaire}

L'utilisation de pièces esquillées - emmanchées ou non comme " outil " intermédiaire pour fracturer, fendre, cliver, fractionner, dégrossir, entailler, creuser, couper, etc., peut s'effectuer en percussion posée ou lancée. En percussion posée, deux catégories : types " coin " et "gouge" (Lindner 1960), peuvent être distinguées en fonction de l'angle (perpendiculaire ou oblique) de travail (LeroiGourhan 1971 p. 58). En percussion lancée ${ }^{8}$ (Rigaud 1977 ; Caspar et al. 1998) deux autres catégories (types " herminette " et " hache "), peuvent également être individualisées en fonction de la fixation (perpendiculaire ou oblique) du " tranchant " par rapport au manche.

(7) enlèvement à ne pas confondre avec un réavivage, certains grattoirs plus ou moins épais s'avérant être des nucléus à lamelles.

(8) comme pour l'usage de certains grattoirs emmanchés observés chez les Sioux (Mason 1891 ; Collin et Jerdón-Giner 1993 p. 116). 

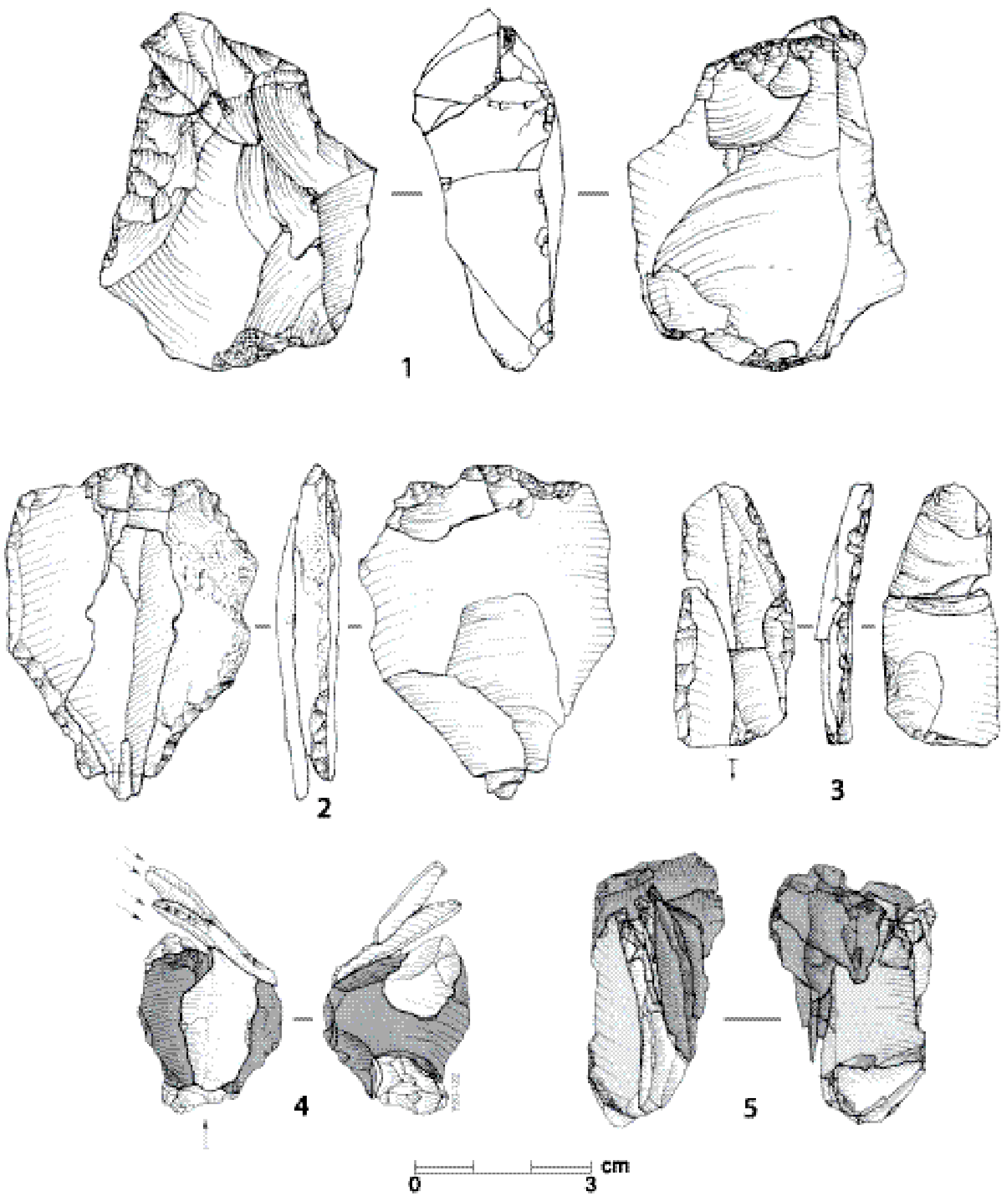

Figure 3 - Exemples de remontages de pièces esquillées. 1, 2 et 3 : Geissenklösterle (d'après Hahn 1988) ; 4 et 5 : HauteriveChampréveyres (d'après Cattin 2002).

Figure 3 - Examples of refit splintered pieces. 1, 2 and 3: Geissenklösterle (after Hahn 1988); 4 and 5: Hauterive-Champréveyres (after Cattin 2002). 

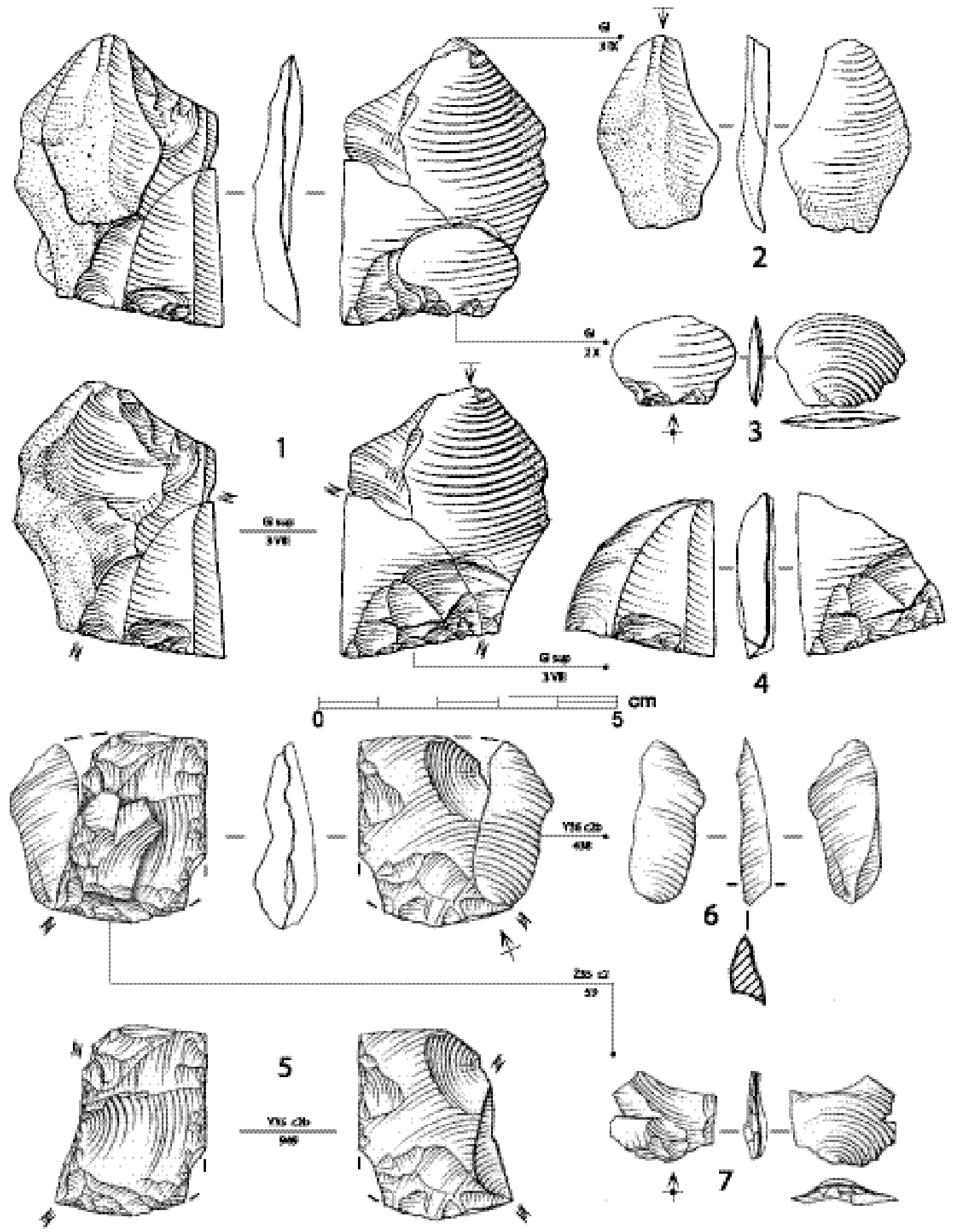

Figure 4 - Exemples de remontages de pièces esquillées de l'Aurignacien ancien (inédits) : 1, 2, 3 et 4 : Le Piage (Fajoles, Lot) ; 5, 6 et 7 : Hui (Beauville, Lot-et-Garonne). Dessins F. Le Brun-Ricalens.

Figure 4 - Examples of refit splintered pieces (Aurignacien I): 1, 2, 3 and 4: Le Piage (Fajoles, Lot); 5, 6 and 7: Hui (Beauville, Lot-et-Garonne). Drawings F. Le Brun-Ricalens. 

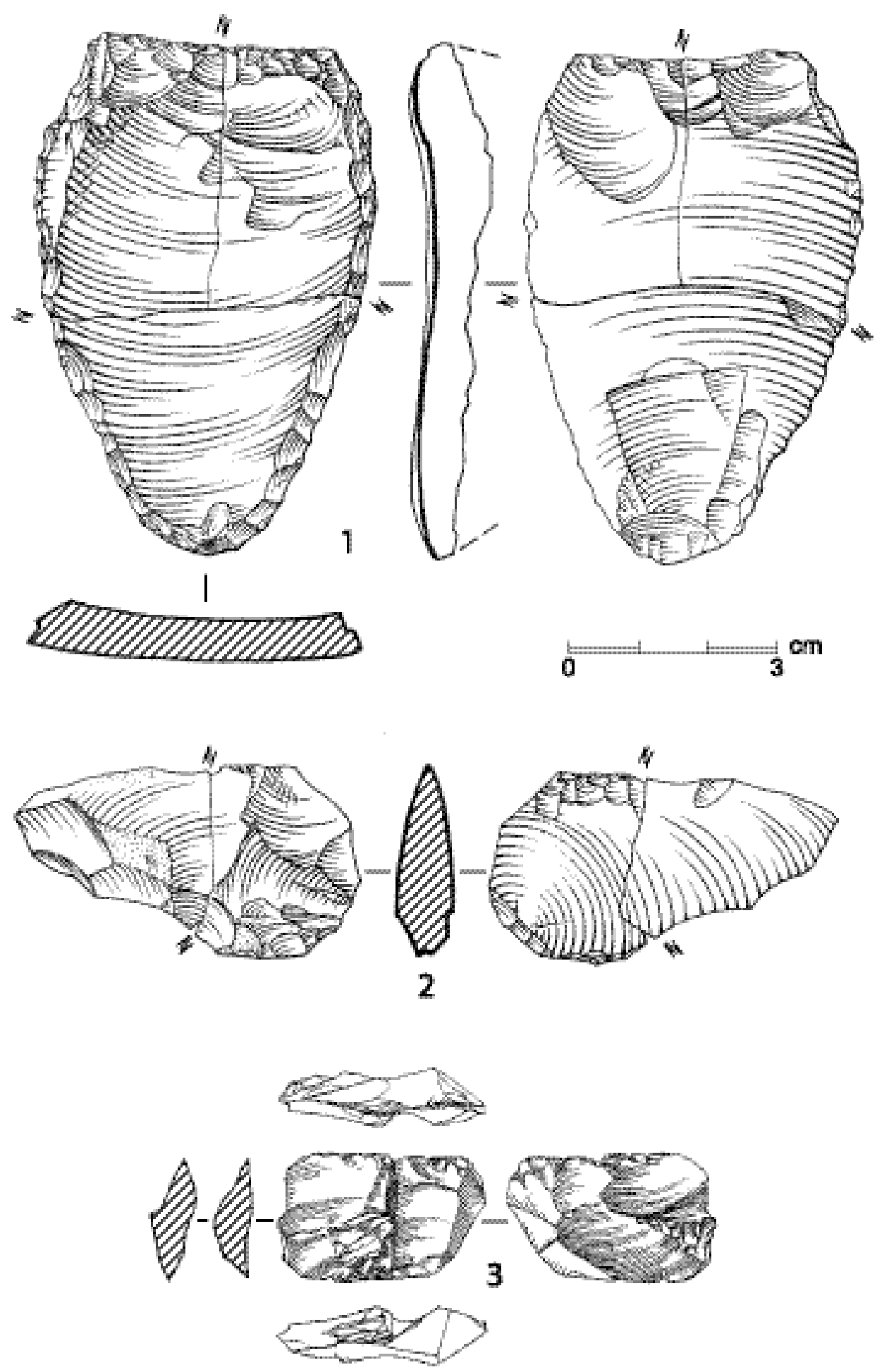

Figure 5 - Exemples de raccords de pièces esquillées à plan de cassure parallèle à l'axe de percusssion de l'Aurignacien ancien (inédits) : 1 : Le Piage (Fajoles, Lot) ; 2 : Hui (Beauville, Lot-et-Garonne) ; 3 : Le Flageolet I (Bézenac, Dordogne). Dessins 1 et 2 : F. Le Brun-Ricalens, $3:$ J.-G. Marcillaud (d'après Lucas et Hays 2004).

Figure 5 - Examples of refit splintered pieces (Aurignacien I): 1, 2, 3 and 4: Le Piage (Fajoles, Lot); 5, 6 and 7: Hui (Beauville, Lot-et-Garonne); 3: Le Flageolet I (Bézenac, Dordogne). Drawings 1 and 2: F. Le Brun-Ricalens ; 3: J.-G. Marcillaud (after Lucas et Hays 2004). 


\subsection{3 - Considérations archéologiques}

Sur le plan archéologique, ces demières décennies, les études de répartition spatiale des industries lithiques et osseuses, ainsi que la pratique systématique des remontages (fig. 3, 4 et 5), permettent de renouveler les approches palethnologiques. Les travaux de cette nature concernant les pièces esquillées sont encore rares. Ces analyses comportementales favorisées notamment par l'examen dynamique des raccords d'esquilles de pièces esquillées, tendent à montrer, au Paléolithique, une utilisation immédiate de ces demières, en particulier à proximité de foyers comme à Hauterive-Champréveyres $(\mathrm{CH})$ (Leesch 1997 ; Cattin 2002). Par ailleurs, sur certains sites aurignaciens comme à Beauville-" Hui " (F), une relation avec certains percuteurs " à cupules " a également été proposée (Le Brun-Ricalens 1989, 1996) mais n'est pas systématique (Perpère et Schmider 2002).

Pour le Paléolithique, ce sont les séries magdaléniennes, grâce à la conservation fréquente des témoins osseux lato sensu (os, bois, dents), qui proposent les documents les plus significatifs pour appréhender certaines techniques de fracturation et de façonnage. Les pièces esquillées de Châleux (B) pourraient avoir servi à fendre des os longs (Dewez 1985), technique identique à celle observée (et obtenue par expérimentation) à l'abri Fritsch (F) pour le clivage de bois de renne à l'aide de " coin " de silex (Allain et al. 1977 ; Averbouh 2000 ; Goutas 2003). Cette technique semble aussi avoir été utilisée pour se procurer de la moëlle (Morin 2007) dans le site éponyme de La Madeleine ( $F)$ pour la fracturation des phalanges de cheval (Bouvier 1979). Le travail de l'ivoire, matériau très résistant, pourrait avoir été débité avec des procédés similaires (Otte 1977 ; Hahn 1995 ; Khlopatchev 2002). Les planches illustrant le site de Brassempouy (F) présentent des " morceaux d'ivoire dégrossis à coups de gouges " (Piette 1907 pl. LXXVI et LXXVII). Dans l'Aurignacien d'Europe occidentale, le double rainurage ne semblant pas usité, la technique de fendage par percussion notamment pour le travail d'ivoire sub-fossile a été proposée (Christensen 1996, 1999 ; White 1996), que cela soit pour la confection d'outils (Leroy-Prost 1975 ; Lollios 1999) ou de sculptures comme la statuette du Hohlenstein-Stadel (D) (Schmid 1989). Par ailleurs, à une échelle plus grande, les proportions différentielles de pièces esquillées constatées dans de nombreuses séries du Paléolithique supérieur nord aquitain $(F)$ pourraient trouver un écho avec les différentes fréquences obsenées de restes de rennes, relation dont la nature reste à expliciter (Demars 2005).

Au Mésolithique, l'emploi de pièce intermédiaire pour fendre a été reconnu parmi les différentes techniques de fracturation des bois de cerf (David 2002). Au Néolithique, le travail de l'os, du bois végétal et animal, connaît un essor non négligeable avec une diversification des outils, en particulier emmanchés pour des activités spécialisées (MacGrégor 1985). Toutefois, les stigmates observés sur les pièces esquillées néolithiques comme celles de Darion, de Liège-" Place St-Lambert", de Blicquy (B) (Cahen et al. 1986 ; Caspar 1988), de Chalain, de Clairvaux et de la
Motte-aux-Magnins (F) (Beugnier 1997), demeurent similaires à ceux rencontrés au Paléolithique et au Mésolithique. Accompagnant le développement des productions agricoles, il a également été envisagé la fabrication de nouveaux outils, en particulier pour dépiquer les céréales (Whallon 1978 ; Ataman 1992 ; Skakun 1992, 1993 ; Anderson et Inizan 1994). Parmi ceux-ci, certains éléments pourraient présenter des similitudes avec les pièces esquillées à l'instar des pièces lithiques insérées par percussion sur un support en bois pour fabriquer des tribulum. Certaines séries néolithiques méditerranéennes étudiées sous cet angle ne confirment pas actuellement cette hypothèse de travail (Gassin 1996 p. 194-195).

\subsection{4 - Considérations ethnographiques}

Sur le plan ethnographique, des artefacts proches des pièces esquillées, appelés "sinews-frayeurs " (effilocheurs de tendons) sont employés en Afrique (Alimen 1955 p. 258) pour fendre des matériaux organiques (animaux ou/et végétaux), notamment pour " diviser les fibres des lianes" (Leakey 1931). A signaler pour une utilisation en percussion lancée, l'importante diversité des montages observables en diverses régions du monde pour l'emmanchement des grattoirs et herminettes (Pétrequin et Pétrequin 1993 ; Brenet et al. 2001).

\subsection{5 - Données expérimentales}

Le travail des matériaux organiques à l'aide de certaines pièces esquillées en percussion directe lancée et indirecte posée (fig. 6) a fait l'objet de différents protocoles expérimentaux (Rigaud 1977 ; Le Brun-Ricalens 1989 p. 198) dans le but de proposer des séries de comparaison pouvant servir de référentiel. À notre connaissance, l'étude actuellement la plus aboutie a été effectuée par G. Lucas et M. Hays. Dans leur contribution publiée en 2004, 48 pièces expérimentales ont été examinées de manière détaillée en les comparant au matériel archéologique aurignacien et périgordien recueilli au Flageolet I. Lors de ces investigations, dans un premier temps ont été fracturés expérimentalement de l'os et du bois, en récoltant, dans la mesure du possible, toutes les esquilles et déchets. Avant et après chaque expérience, chaque artefact a été mesuré et pesé. Ensuite ces artefacts ont fait l'objet de descriptions technotypologiques et d'analyses tracéologiques (27 pièces expérimentales sur 48 et 66 archéologiques) avant d'être classés selon différents critères (dimensions moyennes, matières premières sélectionnées, supports utilisés, poids, emplacement et morphologie des parties actives, types de cassure, etc.). À souligner un apport novateur avec la caractérisation des différentes variétés d'esquilles rencontrées, ainsi que des types de fracture et surfaces de cassure associées (plan de cassure, parallèle ou perpendiculaire, à l'axe de percussion, microcassures avec dièdres). Dans ce travail pionnier, il est proposé d'employer les termes neutres "pôle plan " et "pôle aigu " pour décrire les bords percutés qui permettent de classer les pièces esquillées en trois classes en fonction du nombre d'axes de percussion observé. A l'issue de cette étude, les croisements des diverses données amènent les auteurs à 


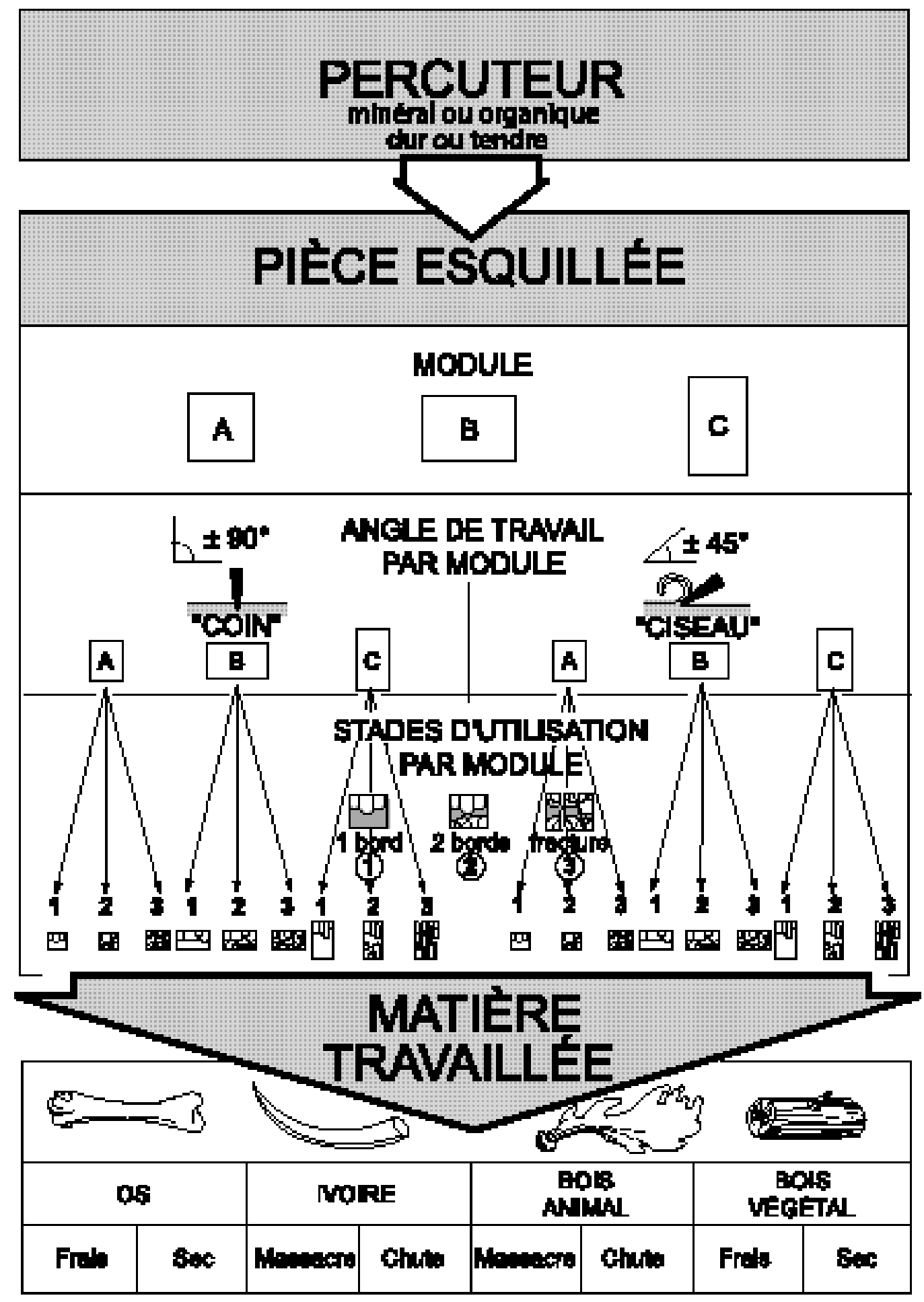

Figure 6 - Schéma expérimental proposé pour tester une des hypothèses de travail relatif à l'emploi de certaines pièces esquillées, à savoir comme " pièce intermédiaire " en percussion indirecte posée pour traiter des matières organiques dures oulet tendres (d'après Le Brun-Ricalens 1989).

Figure 6 - Experimental procedure proposed to test one of the working hypotheses concerning the function of certain splintered pieces, in this case that they served as an "intermediary piece" for the working of hard and/or soft organic materials (after Le Brun-Ricalens 1989). 
se méfier de toute interprétation généralisatrice en rappelant la difficulté de trancher sur la/les fonction(s) des pièces esquillées : "plusieurs processus peuvent aboutir au même résultat " et en invitant à " une analyse spécifique pour chaque site" (Lucas et Hays 2004 p. 119).

Des diverses expérimentations réalisées, il ressort que dans le cadre des techniques de fragmentation, la fracturation longitudinale de matériau organique (os, bois de cervidé, bois végétal, ivoire) effectuée à l'aide d'une pièce esquillée est plus rapide (Camps-Fabrer et d'Anna 1977 ; Lucas et Hays 2004) et efficace, notamment pour extraire la moelle des os longs (Dewez 1985), que le sciage longitudinal avec un burin (Le Brun-Ricalens 1989). Toutefois, ce dernier est plus précis et moins aléatoire. Ces deux techniques ont pu coexister, et peut-être que dans certains cas, certaines pièces esquillées auraient remplacé certains burins (Pradel 1970). Cette hypothèse pourrait en partie expliquer certaines différences régionales observées entre sites de plein air, sous abris et en grottes (Chauchat et al. 1985 ; Le BrunRicalens 1989 ; Demars et Laurent 1992, Demars 2005). Ces différences pourraient exprimer des faciès d'activités particuliers ou/et des choix d'ordre culturel (Binford 1979).

\section{6 - ATTRIBUTION CHRONO-CULTURELLE}

Rares dans le Paléolithique moyen européen, où il ne faut pas confondre les pièces esquillées avec les artefacts présentant des aménagements de type "Kostienki ", les pièces esquillées se rencontrent pendant tout le Paléolithique supérieur - en particulier à l'Aurignacien et au Magdalénien ancien -, mais aussi au Mésolithique, au Néolithique, jusqu'à l'âge du Bronze.

Concemant la gestion des ressources minérales, les proportions de pièces esquillées au sein d'industries semblent refléter à la fois des stratégies " économiques " et opportunistes. Ce type de comportement " économe " vis-à-vis de la matière première est particulièrement perceptible au Néolithique ancien (culture du Rubané) en Europe nordoccidentale dans les régions pauvres en ressources siliceuses où les pièces esquillées sont obtenues essentiellement en fin de chaîne opératoire à partir du recyclage d'outils usés, cassés et autres produits de second choix (Löhr et al. 1977 ; Strien 1984 ; Zimmermann 1988 ; Hauzeur 2003).

\section{7 - BILAN}

\section{Une technique, plusieurs applications}

La grande récurrence des pièces esquillées au sein des industries préhistoriques pléistocènes et holocènes peut s'expliquer par le fait que la technique pour les obtenir est facile à mettre en œuvre. Les pièces esquillées sont géné- ralement issues d'un emploi rapide ${ }^{9}$ nécessitant à la fois peu de savoir-faire et peu d'investissement technique ${ }^{10}$ (Berthelet et Chavaillon 1993). Elles offrent par ailleurs des possibilités et des combinaisons multiples qui en font des artefacts d'appoint appréciés par leur polyvalence, aussi bien dans les sociétés nomades (Bon 2005) que sédentaires. En permettant notamment l'acquisition et le traitement des carcasses animales (confection d'armatures, extraction de la moelle, découpe, etc.) (Patou-Mathis et al. 2005) ou du bois végétal, les pièces esquillées s'avèrent utiles tant dans les sphères d'activités cynégétiques, que domestiques et agricoles.

\section{Les pièces esquillées : outils a posteriori et alii...}

A l'issue de cette enquête, il ressort que pour chacune des hypothèses présentées : utilisation en percussion directe ou indirecte, comme outil, nucléus et pièce intermédiaire, puis discutées à partir d'éléments aussi bien archéologiques, tracéologiques, ethnographiques, qu'expérimentaux, les pièces esquillées présentent in fine de fortes similitudes morphologiques entre elles malgré des emplois différents. En fonction des périodes, des régions, des sites et des activités, ces ressemblances semblent a priori rendre délicate la reconnaissance des utilisations initiales. Toutefois, comme l'ont souligné dans leur conclusion G. Lucas et M. Hays (op. cit.), " la détermination de la fonction des pièces esquillées nécessite par conséquent une analyse spécifique pour chaque site". En effet, en suivant l'exemple des travaux récents réalisés sur les industries du Flageolet I (Lucas et Hays 2004) et de Hauterive-Champréveyres (Leesch 1997 ; Cattin 2002), les intentionnalités peuvent souvent être précisées en croisant notamment les examens lithologiques, technologiques, tracéologiques et typologiques des ensembles lithiques étudiés en général et des divers éléments en relation avec les pièces esquillées en particulier. Pour chaque cas d'étude, il est bienvenu de veiller à valider ces données à l'aide de référentiels expérimentaux.

Afin de continuer à faire avancer la question, il semble nécessaire d'encourager la complémentarité des approches. Dans cette perspective, il est à recommander de les intégrer également aux études de répartition spatiale, avec notamment la prise en compte des informations apportées par les remontages et raccords réalisés au sein des industries lithique et osseuse. Gageons qu'avec le développement en cours d'une véritable dialectique interdisciplinaire, la multiplication des synergies entre les différents protocoles d'études favorisera le renouvellement des points de vue sur les statuts fonctionnels des pièces esquillées. Une telle démarche adaptée à la problématique des pièces esquillées devrait s'avérer pertinente pour entrevoir leurs variabilités et leurs modalités d'utilisation soit comme " nucléus ", " outil " 11 ou/et " pièce intermédiaire " ${ }^{12}$. En l'état actuel des connaissances, en l'absence d'arguments ${ }^{13}$ démontrant un emploi comme " outil " ou

(9) pour une utilisation opportuniste et immédiate ?

(10) car généralement d'une durée d'utilisation très brève dans le temps avec bris fréquent.

(11) au sens typologique : intentionnellement façonné.

(12) générant un outil a posteriori (Bordes 1970b).

(13) à rechercher dans chaque série étudiée. 
" nucléus ", les pièces esquillées sont, par leur technique d'obtention, essentiellement à interpréter comme des " outils a posteriori" (Bordes 1970b).

\section{Remerciements}

II m'est agréable de remercier chaleureusement toutes les personnes qui m'ont prodigué leurs conseils lors de l'élaboration de ce manuscrit, en particulier J. Tixier, H. Floss, J.-G. Bordes, A. Hauzeur, A. Morala, J. Pelegrin, sans oublier les traductions réalisées par M. O'Farrell, C. Szmidt et S. Rick. Le montage infographique des illustrations a bénéficié des talents de J.-N. Anslijn, H.-G. Naton et F. Valotteau.

\section{BIBLIOGRAPHIE}

ALIMEN H. 1955 - Préhistoire de l'Afrique. Ed. Boubée, Paris, $578 \mathrm{p}$.

ALLAIN J. et DESCOUTS J. 1957 - A propos d'une baguette à rainure armée de silex découverte dans le Magdalénien de St Marcel. L'Anthropologie, 61, 5-6, p. 503-512.

ALLAIN J., FRITSCH R., RIGAUD A. et TROTTIGNON F. 1977 - Le débitage du bois de renne dans les niveaux à raclettes du Badegoulien de l'abri Fritsch et de sa signification. In : Université de Provence (Ed), Premier colloque international sur l'industrie de l'os dans la Préhistoire, Sénanque (1974), p. 67-72.

ANDERSON P. C. et INIZAN M.-L. 1994 - Utilisation du tribulum au début du IIIème millénaire : des lames " caca néennes " lustrées à Kutan (Ninive $V$ ) dans la région de Mossoul, Iraq. Paléorient, 20, 2 p. 85-104.

ATAMAN K. 1992 - Threshing sledge and archaeology. In : P. C. Anderson (Ed.), Préhistoire de l'agriculture. Monographie du CRA, 6, CNRS Éd., Paris, p. 305-320.

AUBRY TH., FONTUGNE M. et MOURA M.-H. 1997 - Les occupations de la grotte de Buraca Grande depuis le Paléolithique supérieur et les apports de la séquence holocène à l'étude de la transition Mésolithique/Néolithique au Portugal. Bulletin de la Société Préhistorique Française, 94,2 , p. $182-190$.

AUBRY TH., ZILHÃO J., ALMEIDA F. et FONTUGNE M. 1998 - Production d'armatures microlithiques pendant le Paléolithique supérieur et le Mésolithique au Portugal. In: Balbín Behrmann, R.; Buena Ramírez, P. (eds) - I/ Congresso Peninsular de Arqueologia Peninsular. Zamora (24-27 septembre 1996) Tomo I - Paleolítico y Epipaleolítico. Zamora: Fundación Rei Afonso Henriques, p. 259-272.

AVERBOUH A. 2000 - Technologie de la matière osseuse travaillée et implications palethnologiques; l'exemple des chaînes d'exploitation du bois de cervidé chez les
Magdaléniens des Pyrénées. Université de Paris I, 2000. 2t. $500 \mathrm{p}$.

BARDON, L. et BOUYSSONIE, A. et J. 1906 - Outils écaillés par percussion à la Coumba-del-Bouittou, près Brive (Corrèze). Revue de l'école d'Anthropologie de Paris, 16, 2, p. 170-175.

BARDON L. et BOUYSSONIE A. et J. 1908 - Station de la Coumba-del-Bouïtou, près Brive (Corrèze). Bulletin de la Société scientifique, historique et archéologique de la Corrèze (1907), $54 \mathrm{p}$.

BARHAM L. 1987 - The bipolar technique in Southern Africa - a replication experiment. South African Archaeological Bulletin, 42, p. 45-50.

BERTHELET A. et CHAVAILLON J. (Eds) 1993 - The use of tools by human and non-human primates. Symposium de la fondation Fyssen, Oxford, Clarendon Press, 1 vol. 424 p.

BEUGNIER V. 1997 - L'usage du silex dans l'acquisition et le traitement des matières animales dans le Néolithique de Chalain et Clairvaux. Thèse de Doctorat de l'Université de Paris X-Nanterre, $491 \mathrm{p}$.

BINDER D. 1987 - Le Néolithique ancien provençal : technologie et typologie des outillages lithiques. Supplément à Gallia-Préhistoire, CNRS éd., Paris, 24, 205 p.

BINFORD L. R. 1979 - Organization and formation prozesses: looking at curated technologies. Journ. Anthr. Research, 35, p. 255-273.

BON F. 2005 - Little big tool. Enquête autour du succès de la lamelle. In : Le Brun-Ricalens F., Bordes J.-G. et Bon F. (coord.) Productions lamellaires attribuées à l'Aurignacien : chaînes opératoires et perspectives technoculturelles. Acte du XIV congrès de l'Union Internationale des Sciences Pré- et Protohistoriques de Liège (2-8 septembre 2001), ArchéoLogiques 1, Musée national d'Histoire et d'Art, Luxembourg, p. 479-484.

BORDES F. 1961 - Typologie du Paléolithique ancien et moyen. Publications de l'Institut de Préhistoire de l'Université de Bordeaux, mémoire 1, 2 vol. 103 p.

BORDES F. 1967 - Considérations sur la Typologie et les techniques dans le Paléolithique. Quartär, 18, p. 25-55.

BORDES F. 1970a - Observations typologiques et techniques sur le Périgordien supérieur de Corbiac (Dordogne). Bulletin de la Société Préhistorique Française, C. R. S. M., 67 , p. $105-113$.

BORDES F. 1970b - Réflexions sur l'outil au Paléolithique. Bulletin de la Société Préhistorique Française, C. R. S. M., 67 , p. $199-202$.

BOUVIER J.-M. 1979 - Le mystère des fendeurs de phalanges ou contribution à la connaissance du travail de l'os 
au Magdalénien IV. Bulletin de la Société Préhistorique Française, 76, 4, p. 105-109.

BRENET M., SANCHEZ-PRIEGO J., IBANEZ-ESTEVEZ J. J. 2001 - Les pierres de construction taillées en calcaire et les herminettes en silex du PPNA de Jerf El Ahmar (Syrie), analyses technologique et expérimentale. In : Bourguignon L., Ortega I., Frère-Sautot M.-C. (dir.) - Préhistoire et approche expérimentale. Préhistoires 5, éd. M. Mergoil, p. 121-164.

BREUIL H. 1932 - Le feu et l'industrie de pierre et d'os dans le gisement du "Sinanthropus" à Chou-kou-tien. L'Anthropologie, 42, p. 1-17.

BRÉZILLON M. 1968 - La dénomination des objets de pierre taillée. Matériaux pour un vocabulaire des préhistoriens de langue française. IVème supplément à GalliaPréhistoire C.N.R.S. édition, 416 p.

CAHEN D., CASPAR J.-P. et OTTE M. 1986 - Industries lithiques danubiennes de Belgique. ERAUL 21, 88 p.

CAMPS-FABRER H. et D'ANNA A. 1977 - Fabrication expérimentale d'outils à partir des métapodes de mouton et de tibias de lapin. In : H. Camps-Fabrer (éd.), Méthodologie appliquée à l'industrie de l'os préhistorique. Colloques internationaux du CNRS $n^{\circ} 568$, Abbaye de Sénanque (Vaucluse) (9-12 juin 1976) CNRS éd., Paris, p. 311-326.

CANCELLIERI E., COPPOLA A., D'ANGEL E., DI LEO R., RINALDI M. F., ROSSI D. 2001 - La tecnica di scheggiatura bipolare applicata ai ciottoli. Considerazioni su un lavoro di scheggiatura sperimentale. In : Cavarretta G., Gioia P., Mussi M., Palombo M. P. (eds) - La Terra degli Elefanti - The world of Elephants, Atti del $1^{\circ}$ Congresso Internazionale, Roma (16-20 ottobre 2001) CNR, Roma, p. 23-26.

CASPAR J.-P. 1988 - Contribution à la tracéologie de l'industrie lithique du Néolithique ancien de l'Europe nordoccidentale. Dissertation, Université catholique de Louvain-la-Neuve, 2 vol. 194 p.

CASPAR J.-P., BURNEZ-LANOTTE L. et ROTS V. 1998 Le grattoir herminette dans le groupe de Blicquy : approche expérimentale. Inteméo 2, Paris, p. 39-40.

CATTIN M.-I. 2002 - Un campement magdalénien au bord du lac de Neuchâtel. Exploitation du silex (secteur 1). Hauterive-Champréveyres 13, Archéologie neuchâteloise 26, tome 1, $262 \mathrm{p}$.

CHAUCHAT CL., NORMAND C., RAYNAL J.-P. et SANTAMARIA R. 1985 - Le retour de la pièce esquillée. Bulletin de la Société Préhistorique Française, 82, 2, p. 35-41.

CHEYNIER A. 1934 - Les lamelles à bord abattu et les pièces microlithiques dans le Solutréen final de Badegoule. Bulletin de la Société Préhistorique Française, 31, p. 291-305.
CHEYNIER A. 1963 - La caverne de Pair-non-Pair, Gironde. Documents d'Aquitaine, 2. Bordeaux, 219 p.

CHRISTENSEN M. 1996 - Un exemple du travail de l'ivoire du paléolithique allemand : apport de l'étude fonctionnelle des outils aurignaciens de Geissenklösterle. Techne, 3, p. 39-53, 14 fig.

CHRISTENSEN M. 1999 - Technologie de l'ivoire au Paléolithique supérieur. Caractérisation physico-chimique du matériau et analyse fonctionnelle des outils de transformation. BAR International Series 751, 201 p.

COLLIN F. et JARDÓN-GINER P. 1993 - Travail de la peau avec des grattoirs emmanchés. Réflexions sur des bases expérimentales et ethnographiques. In : Anderson P. C., Beyries S., Otte M., Plisson H. (dir.) Traces et Fonction : les gestes retrouvés. Actes du Colloque international de Liège (8-10 décembre 1990). ERAUL 50, p. 105-117.

COUTIER L. 1929 - Expériences de taille pour rechercher les anciennes techniques paléolithiques. Bulletin de la Société Préhistorique Française, 26, p. 172-174.

CRÉMILLEUX H. et LIVACHE, M. 1976 - Pour le classement des pièces écaillés. Dialektikê, Cahiers de typologie analytique, p. 1-5.

CRESSON J.-H. 1977 - Reply to: The myth of bipolar flaking industries by L.W. Patterson and J.B. Sollberger (1976). Lithic Technology, 6, 3, p. 27.

D'ANGELO E. et MUSSI M. 2005 - Galets et lamelles de l'Aurignacien du Latium (Italie centrale) : le cas de la grotta Barbara. In : Le Brun-Ricalens F., Bordes J.-G. et Bon F. (coord.) P roductions lamellaires attribuées à l'Aurignacien : chaînes opératoires et perspectives technoculturelles. Acte du XIV ${ }^{\mathrm{e}}$ congrès de l'Union Internationale des Sciences Pré- et Protohistoriques de Liège (2-8 septembre 2001), ArchéoLogiques 1, Musée national d'Histoire et d'Art, Luxembourg, p. 313-322.

DAVID E. 2002 - L'industrie en matières dures animales du Mésolithique ancien et moyen en Europe du Nord : contribution de l'analyse technologique à la définition du Maglemosien. Thèse de doctorat. Université de Paris XNanterre.

DEMARS P.-Y. 1986 - L'interprétation des industries lithiques et leur rapports avec la culture. Centre de recherches Préhistoriques, 10, p. 87-93.

DEMARS P.-Y. 2005 - La structuration de l'espace chez les chasseurs-cueilleurs du Paléolithique supérieur dans le Nord de l'Aquitaine. In : Jaubert J., Barbaza M. (dir.), Territoires, déplacements, mobilité, échanges durant la Préhistoire Terres et hommes du Sud. Actes du 126 ème Congrès national des Sociétés Historiques et Scientifiques, Toulouse (9-14 avril 2001), éd. du CTHS, p. 149-159. 
DEMARS P.-Y. et LAURENT P. 1992 - Types d'outils lithiques du Paléolithique supérieur en Europe. Presses du C.N.R.S. 178 p.

DEWEZ M. 1985 - Les pièces esquillées dans le Paléolithique supérieur de Belgique. Bulletin de la Société Préhistorique Française, 82, 5, p. 131-133.

EICKHOFF S. 1988 - Ausgesplitterte Stücke, KostienkiEnden und „retuschierte Bruchkanten“. Einige Aspekte zur Untersuchungen der Artefakte aus westeuropäischem Feuerstein auf dem Magdalénien-Fundplatz Gönnersdorf. Arch. Inf. 11, p. 136-144.

ESCALON DE FONTON M. 1969 - La pièce esquillée, essai d'interprétation. Bulletin de la Société Préhistorique Française, 66, p. 76.

FIEDLER L. 1979 - Formen und Techniken neolithischer Steingeräte im Rheinland. Beiträge zur Urgeschichte des Rheinlandes III. Rheinische Ausgrabungen, 19, p. 53-190.

FRISON G. C. 1988 - Paleoindian subsistence and settlement during Post-Clovis times of the northwestem plains. The adjacent moutain ranges, and intermontane basins. In : Carlisle R. C. (eds) Americans before Colombus : Ice-Age Origins. Departement of Anthropology, Ethnology Monograph 12. University of Pittsburgh.

GASSIN B. 1996 - Evolution socio-économique dans le chasséen de la grotte de l'église supérieure (Var). Monographie du C.R.A. 17.

GOBERT E.-G. 1958 - Er Recheda es Souda ou Presqu'île 26. Karthago, IX, p. 1-44.

GOUTAS N. 2003 - Identification de deux procédés de débitage inédits du bois de cervidés dans les niveaux gravettiens de Laugerie-haute Est et Ouest. Paléo , 15, p. 255-262.

GRAMLY R.M. 1990 - Guide to the palaeo-Indian artifacts of North America. Buffalo society of natural sciences, occasional paper $n^{\circ} 4$, Buffalo NY, Persimmon Press, 1 vol. 78 p.

GUYODO J.-N., MARCHAND G. 2005 - La percussion bipolaire sur enclume dans l'Ouest de la France de la fin du Paléolithique au Chalcolithique : une lecture économique et sociale. Bulletin de la Société Préhistorique Française, 102, 3, p. 539-549.

HAHN J. 1977 - Aurignacien. Das ältere Jungpaläolithikum in Mittel- und Osteuropa. Fundamenta A9, Köln-Graz.

HAHN J. 1988 - Das Gessenklösterle-Höhle im Achtal bei Blauberen 1. Fundhorizontbildung und Besiedlung im Mittlepaläolithikum und im Aurignacien, Konrad Theiss Verlag, Stuttgart, Forschungen und Berichte zur vor-und Frühgeschichte im Baden-Würtemberg, 26, 262 p.

HAHN J. 1993 - Erkennen und Bestimmen von Stein- und Knochenartefakten. Einführung in die Artefaktmorphologie.
Verlag Archaeologia Venatoria, Bd. 10, Institut für Urgeschichte der Universität Tübingen, 398 p.

HAHN J. 1995 - Les ivoires en Allemagne, débitage, façonnage et utilisation au Paléolithique supérieur. In : Hahn et al. (éd.). Le travail et l'usage de l'ivoire au Paléolithique supérieur. Actes de la table ronde de Ravello (29-31 mai 1992) Rome, p. 115-132.

HAMY E. T. 1906 - Matériaux pour servir à l'Histoire de l'archéologie préhistorique. I) Le mémoire de Mahudel sur les pierres de foudre (1730). Revue archéologique, p. 239-259.

HAUZEUR A. 2003 - Contribution à l'étude du Rubané du Nord-Ouest : sites du Grand-Duché de Luxembourg en bassin mosellan. Thèse de Doctorat européen des Universités de Strasbourg et de Liège, 2 vol., 333 p. et 317 p.

HAYDEN B. 1980 - Confusion in the bipolar world : bashed pebbles and splintered pieces. Lithic technology, 9, 1, p. 2-27.

HAYNES G. 1977 - Reply to: The myth of bipolar flaking industries by Sollberger and Patterson. Lithic technology, $6,1-2$, p. 5 .

HAYS M. et LUCAS G. 2001 - Experimental investigations of Aurignacian bladelets. In : M. Hays, P. Thacker (dir.), Questionning the answers: resolving fundamental problems of the Early Upper Paleolithic. British Archaeological Reports, Oxford, p. 109-116.

HEINZELIN DE BRAUCOURT J. de 1962 - Manuel de typologie des industries lithiques. Bruxelles, $74 \mathrm{p}$.

INIZAN M.-L., REDURON M., ROCHE H. et TIXIER J. 1995 - Technologie de la pierre taillée. Tome 4, éd. C.R.E.P., 199 p.

JONES P. 1994 - Results of experimental work in relation to stone industries of Olduvai Gorge. In : M.D. Leakey and D. Roe (Eds) Olduvai Gorge volume 5, Cambridge University Press, p. 254-298.

KAMMINGA J. 1971 - Microscopic and experimental study of Australian Aboriginal stone tools. University of Sydney (unpublished).

KEELEY L. H. 1980 - Experimental determination of stone tools uses. A microwear analysis. University of Chicago Press.

KHLOPATCHEV G. 2002 - Les techniques de débitage de l'ivoire dans les sites de la plaine russe au Paléolithique supérieur. Préhistoire Européenne, 16-17, p. 215-230.

LEAKEY L. 1931 - The Stone age Culture of Kenya. Cambridge University Press.

LE BRUN-RICALENS F. 1989 - Contribution à l'étude des pièces esquillées : la présence de percuteurs à "cupules". Correspondance scientifique. Bulletin de la Société Préhistorique Française, 86, 7,1p. 96-200. 
LE BRUN-RICALENS F. 1996 - L'occupation d'Hui à Beauville, Lot-et-Garonne. Activités domestiques en plein air à l'Aurignacien ancien, In : Société préhistorique française et Faton (Eds), La Vie préhistorique, p. 80-85.

LE BRUN-RICALENS F. 2005 - Chronique d'une reconnaissance attendue. Outils " carénés", outils " nucléiformes ": nucléus à lamelles. Bilan après un siècle de recherches typologiques, technologiques et tracéologiques. In : Le Brun-Ricalens F., Bordes J.-G. et Bon F. (coord.) P roductions lamellaires attribuées à l'Aurignacien : chaînes opératoires et perspectives technoculturelles. Acte du XIVE congrès de I'Union Internationale des Sciences Pré- et Protohistoriques de Liège (2-8 septembre 2001), ArchéoLogiques 1, Musée national d'Histoire et d'Art, Luxembourg, p. 23-72.

LEESCH D. 1997 - Hauterive-Champréveyres. 10. Un campement magdalénien au bord du lac de Neuchâtel : cadre chronologique et culture, mobilier et structures, analyse spatiale (secteur 1). Musée cantonal d'archéologie, Neuchâtel. Archéologie neuchâteloise, 19, 270 p.

LENOIR M. 1975 - Style et technologie lithique. Bulletin de la Société Préhistorique Française, 72, 2, p. 46-49.

LEROI-GOURHAN A. 1971 - Évolution et technique. L'homme et la matière. Collection Sciences d'Aujourd'hui, Albin Michel, Paris (2 ${ }^{\text {nd }}$ éd.), 475 p.

LEROY-PROST Chr. 1975 - L'industrie osseuse aurignacienne, essai régional de classification : Poitou, Charente, Périgord. Gallia-Préhistoire, 18, 1, p. 75-156.

LINDNER H. 1960 - Hohlmeißeilgeräte. Steinzeitfragen der Alten und Neuen Welt. Festschr. L. F. Zotz, p. 263-270.

LIOLLIOS D. 1999 - Variabilité et caractéristiques du travail des matières osseuses au début de l'Aurignacien : approche technologique et économique. Université de Paris X, $362 \mathrm{p}$.

LÖHR H. et ZIMMERMANN A. 1977 - Feuersteinartefakte. In : Kuper R. et al. (éd.). Der bandkeramische Siedlungplatz Langweiler 9. Gemeinde Aldenhoven, Kreis Düren. Bonn, Rheinland Verlag, Rheinische Ausgrabungen, 18, 1, p. 131266.

LOTHROP J. C. 1982 - Pièces esquillées from the Vail site. Archaeology of Eastern North America , 10, p. 1-22.

LUCAS G., et HAYS M. 2004 - Les pièces esquillées du site paléolithique du Flageolet I (Dordogne) : outils ou nucléus ? In : Approches fonctionnelles en Préhistoire. XXVème Congrès Préhistorique de France (24-26 novembre 2000) Nanterre, p. 107-120.

LWOFF S. 1967 - Ciseaux à facettes et ciseaux à chanfrein de la grotte de la Marche (Lussac-les-Châteaux, Vienne). Bulletin de la Société Préhistorique Française, 66, 3, p.124-127.
MACDONALD G. F. 1968 - Debert, : A Palaeo-Indian Site in Central Nova Scotia. National Museum of Canada Anthropology paper 16, Ottawa, Ontario, Canada.

MACGREGOR A. 1985 - Bone, Antler, Ivory \& Horn. The Technology of Skeletal Materials Since the Roman Period. B a mes \& Noble Books, Totowa, New Jersey, 245 p.

MAHUDEL 1730 - Sur les prétendues pierres de foudre. Académie des Inscriptions et Belles-Lettres (voir Hamy, 1906).

MANSUR-FRANCHOMME M. E. 1986 - Microscopie du matériel lithique préhistorique. Traces d'utilisation, altérations naturelles, accidentelles et technologiques. Exemples de Patagonie. Cahier du Quaternaire, 9, CNRS éd., Paris, 286 p.

MASON O. T. 1891 - Aboriginal Skin-Dressing. Smithsonian Annual Report.

MAZIĖRE G. 1984 - La pièce esquillée, outil ou déchet ? Bulletin de la Société Préhistorique Française, 81, 6, p. 182-187.

MIGAL W. 1987 - Morphology of splintered piece in the light of experimental method. Arch. Interreg. New in Stone age Archaeology, p. 9-33.

MORIN E. 2007 - Fat composition and Nunamiut decisionmaking: a new look at the marrow and bone grease indices. Journal of Archaeology Science, 34, p. 69-82.

MÜLLER-BECK H. 1957-58 - Zur Bezeichnung paläolithischer Artefakttypen. Alt-Thüringen 143 p.

NEWCOMER M. H. et HIVERNEL-GUERRE F. 1974 Nucléus sur éclat : technologie et utilisation par différentes cultures préhistoriques. Bulletin de la Société Préhistorique Française, 71, 4, p. 119-128.

NUZHNUYJ D. 1989 - L'utilisation des microlithes géométriques et non géométriques comme armatures de projectiles. Bulletin de la Société Préhistorique Française, 86, 3, p. 88-96.

NELISSEN A. 1956 : Signalisation d'un grattoir particulier du Néolithique du faciès de Michelsberg. Bulletin de la Société Préhistorique Française, 53, 49.

OCTOBON E. 1938 - Contribution à l'étude des outillages, ciseaux et pièces esquillées. Bulletin de la Société Préhistorique Française, 35, p. 409-412

ORLIAC E. et ORLIAC M 1973 - La succession des industries à la grotte de la Tourasse (Saint-Martory, HauteGaronne). Bulletin de la Société Préhistorique Française, 70, 3, p. 66-68.

OTTE M. 1977 - Observations sur le débitage et le façonnage de l'ivoire dans l'Aurignacien en Belgique. In : 
Premier colloque international sur l'industrie de l'os dans la Préhistoire, Sénanque 1974, Université de Provence éd., p. 93-96.

PATOU-MATHIS M., PÉAN S., VERCOUTÈRE C., AUGUSTE P. et LAZNICKOVA-GONYSEVOVA M. 2005 Réflexions à propos de l'acquisition et de la gestion de matières premières animales au Paléolithique. Exemples : mammouth/ivoire - renne/bois. In : D. Vialou, J. Renault-Miskovsky, Patou-Mathis M. (dir.), Comportements des hommes du Paléolithique moyen et supérieur en Europe : territoires et milieux. Actes du colloque du G.D.R. 1945 du CNRS, Paris, 8-10 janvier 2003. Liège, ERAUL 111, p. 27-38.

PATTERSON L.W. et SOLLBERGER J.B. 1976 - The myth of bipolar flaking industries. Lithic Technology, 5, 3, p. $40-42$.

PELEGRIN J. 1986 - Technologie lithique : une méthode appliquée à l'étude de deux séries du Périgordien ancien - Roc-de-Combe, couche 8 et La Côte, niveau III. Thèse de doctorat, Université de Paris X-Nanterre, 584 p.

PELEGRIN J. 1988 - esquillé, esquillement, In : (A., Leroi-Gourhan éd.) Dictionnaire de la Préhistoire. Presses Universitaires de France, Paris, 367.

PELEGRIN J. 2000 - Les techniques de débitage laminaire au Tardiglaciaire : critères de diagnose et quelques réflexions. In : L'Europe centrale et septentrionale au Tardiglaciaire. Actes de la table-ronde de Nemours (1316 mai 1997). éd. A.P.R.A.I.F. Mémoires du Musée de Préhistoire d'île de France, 7, p. 73-86.

PERPÈRE M. et SCHMIDER B. 2002 - L'outillage lithique. In : Schmider (dir.) - L'Aurignacien de la grotte du Renne. Les fouilles d'André Leroi-Gourhan à Arcysur-Cure (Yonne). XXXIVeme supplément à GalliaPréhistoire, p. 143-195.

PÉTREQUIN A.-M. et PÉTREQUIN, P. 1993 - Ecologie d'un outil : la hache de pierre en Irian Jaya (Indonésie). Monographie du C.R.A. 12, CNRS, Paris, 439 p.

PIETTE E. 1907 - L'Art pendant l'âge du Renne. 2 vol., Masson éd. Paris.

PLISSON H. et VAUGHAN P. 2002 - Tracéologie. In : Cattin M.-I. (dir.) - Un campement magdalénien au bord du lac de Neuchâtel. Exploitation du silex (secteur 1). Hauterive-Champréveyres 13, Archéologie neuchâteloise 26, tome 1, 262 p. 90-105.

PRADEL L. 1970 - Pièces à bord fracturé et aminci du Paléolithique supérieur. Bulletin de la Société Préhistorique Française, 67, 3, p. 79-80.

RIGAUD A. 1977 - Analyses typologiques et technologiques des grattoirs magdaléniens de la Garenne à StMarcel (Indre). Gallia-Préhistoire, 20, p. 25-43.
RODRIGUEZ A. à paraitre - L'analyse fonctionnelle de l'industrie lithique du gisement épipaléolithique/mésolithique d'El Roc de Migdia (Catalogne, Espagne). Résultats préliminaires.

ROZOY J.-G. 1968 - L'étude du matériel brut et des microburins dans l'Epipaléolithique (Mésolithique) franco-belge. Bulletin de la Société Préhistorique Française, 65, 1, p. 365-390.

ROZOY J.-G. 1978 - Les derniers chasseurs. Bulletin de la société archéologique champenoise, 3 tomes.

RUSSEL-STAFFORD C. 1977 - Reply to: The myth of bipolar flaking industries by L.W. Patterson and J.B. Sollberger (1976). Lithic Technology, 6, 3, p. 27-28.

RUST A. 1943 - Die Alt-und mittelsteinzeitlichen Funde von Stellmoor. Karl-Wachholtz Verlag, Neumünster.

SAUMAGNE P. 1957 - Essai d'explication de l'utilisation de trois silex énigmatiques. Bulletin de la Société Préhistorique Française, 54, p. 470-471.

SCHMID E. 1989 - Die Altsteinzeitliche Elfenbeinstatuette aus der Höhle Stadel im Hohlenstein bei Asselfingen, AlbDonau Kreis. In : Fundberichte aus Baden-Württemberg, Bd. 14, Stuttgart, p. 33-118.

SCHOTT M. 1989 - Bipolar Industries : Ethnographic Evidence and Archaeological Implications,. North American Archaeologist, 10, 1, p. 1-24.

SCHOTT M. 1999 - On bipolar Reduction and Splintered Pieces. North American Archaeologist, 20, p. 217-238.

SEMENOV S. A. 1964 - P rehistoric technology ; An experimental Study of the Oldest Tools and Artefacts from Traces of Manufactures and Wear. London. Cory, Adams et Mackay, $211 \mathrm{p}$.

SKAKUN N. N. 1992 - Évolution des techniques agricoles en Bulgarie chalcolithique (d'après les analyses tracéologiques). In : P. C. Anderson (éd.), Préhistoire de l'Agriculture Monographie du CRA, 6, CNRS éd., Paris, $p$. 289-304.

SKAKUN N. N. 1993 - Agricultural implements in Neolithic and Eneolithic cultures of Bulgaria. In P. C. Anderson, S. Beyries, M. Otte, H. Plisson (éd.), Traces et fonction : les gestes retrouvés. Actes du Colloque international de Liège (8-10 décembre 1990), ERAUL 50, p. 477-488.

SONNEVILLE-BORDES D. DE, et PERROT J. 1953 - Essai d'adaptation des méthodes statistiques au Paléolithique supérieur. Premiers résultats. Bulletin de la Société Préhistorique Française, 50, p. 323-333.

SONNEVILLE-BORDES D. DE, et PERROT J. 1956 Lexique typologique du Paléolithique supérieur : outillage lithique. V. Outillage à bord abattu. VI. Pièces tronquées. 
VII. Lames retouchées. VIII. Pièces variées. IX. Outillage lamellaire Pointe azilienne. Bulletin de la Société Préhistorique Française, 53, p. 547-559.

STRIEN H.-C. 1984 - Die bandkeramische Siedlung Stuttgart-Möhringen 6. Diplôme de Maîtrise de l'Université de Tübingen.

SYMENS N. 1988 - Gebrauchspuren der Steinartefakte. In : J. Hahn (dir.) - Die Geißenklösterle-Höhle im Achtal bei Blaubeuren I. Forsch. U. Ber. Zur Vor- u. Frühgesch. Bad.-Württ. 26, Stuttgart.

TAUTE W. 1968 - Die Stielspitzen-Gruppen im nördlichen Mitteleuropa. Fundamenta A/5, Köln-Graz.

TIXIER J. 1954 - Le gisement préhistorique d'El Hammel. Libyca, 2, p. 79-120.

TIXIER J. 1963 - Typologie de l'Epipaléolithique du Maghreb. Mémoire du centre de recherches anthropologiques, préhistoriques et ethnographiques, 2, Alger, Paris A.M.G., 212 p.

TIXIER J., INIZAN M.-L., et ROCHE H. 1980 - Préhistoire de la pierre taillée, vol.1, Terminologie et technologie. Ed. C.R.E.P., 120 p.

TIXIER J. et TURQ A. 1999 - Kombewa et alii. Paléo, 11, p. $135-143$

TRINGHAM R., COOPER G., ODELL G., VOYTEK B., WHITMAN A. 1974 - Experimentation in the formation of Edge Damage: a new approach to Lithic Analysis. Journal of Field Archaeology, 1, p. 171-196.
VAUGHAN P. 1985a - Funktionsbestimmungen von Steingeräten anhand mikroskopischer Gebrauchspuren. Germania, 63, p. 309-329.

VAUGHAN P. 1985b - Use-wear analysis of flaked stone tools. University of Arizona Press. 204 p.

VILLA P., DELAGNES A. et WADLEY L. 2005 - A late Middle Stone Age artefact assemblage from Sibudu (KwaZulu-Natal) : comparisons with the European Middle Paleolithic. Journal of Archaeological Science, 32 , p. 399-422.

WHALLON R. J. 1978 - Treshing sledge flints. A distinctive pattem of wear. Paléorient, 4, p. 319-324.

WHITE J. P. 1968 - Fabricators, outils écaillés or scalar cores? Mankind, 6, p. 658-666.

WHITE J. P. 1977 - Reply to: The myth of bipolar flaking industries by L.W. Patterson and J.B. Sollberger (1976). Lithic Technology, 6, 1-2, p. 6.

WHITE R. 1996 - Actes de subsistance : de la matière au sens dans la représentation paléolithique. Techne, 3, p. 29-38.

ZILHÃO J., AUBRY TH. et ALMEIDA F. 1997 - L'utilisation du quartz pendant la transition Gravettien-Solutréen au Portugal. Préhistoire Anthropologie Méditerranéennes, LAPMO, Université de Provence, 6, p. 289-303.

ZIMMERMANN A. 1988 - Steine. In : Boelicke U. et al. (éd.). Der bandkeramische Siedlungplatz Langweiler 8. Gemeinde Aldenhoven, Kreis Düren. Bonn, Rheinland Verlag, Rheinische Ausgrabungen, 28, 2, p. 569-787. 
